MZ-TH/05-23

\title{
A Minimal Length from the Cutoff Modes in Asymptotically Safe Quantum Gravity
}

\author{
Martin Reuter and Jan-Markus Schwindt* \\ Institute of Physics, University of Mainz, \\ Staudingerweg 7, D-55128 Mainz, Germany
}

\begin{abstract}
Within asymptotically safe Quantum Einstein Gravity (QEG), the quantum 4-sphere is discussed as a specific example of a fractal spacetime manifold. The relation between the infrared cutoff built into the effective average action and the corresponding coarse graining scale is investigated. Analyzing the properties of the pertinent cutoff modes, the possibility that QEG generates a minimal length scale dynamically is explored. While there exists no minimal proper length, the QEG sphere appears to be "fuzzy" in the sense that there is a minimal angular separation below which two points cannot be resolved by the cutoff modes.
\end{abstract}

\footnotetext{
*E-mail: reuter@thep.physik.uni-mainz.de, schwindt@thep.physik.uni-mainz.de
} 


\section{Introduction}

It is an old speculation, based upon various heuristic arguments [1, that quantum gravity induces a lower bound on physically realized distances. Since this issue can be addressed only in a fundamental quantum theory of gravity (as opposed to a low energy effective theory) it is natural to analyze it within Quantum Einstein Gravity (QEG). This theory is an attempt at the nonperturbative construction of a predictive quantum field theory of the metric tensor by means of a non-Gaussian renormalization group $(\mathrm{RG})$ fixed point [2]-[19]. From what is known today it appears indeed increasingly likely that there does exist an appropriate fixed point which renders QEG nonperturbatively renormalizable or "asymptotically safe" [10, 5, 7, 8,

An important tool in analyzing the RG flow of QEG is the effective average action and its exact functional RG equation [20, 21]. In the case of QEG [3], the average action is a diffeomorphism invariant functional of the metric, $\Gamma_{k}\left[g_{\mu \nu}\right]$, which depends on a variable infrared (IR) cutoff $k$. For $k \rightarrow \infty$ it approaches the bare action $S$, while it equals the standard effective action at $k=0$. The effective field equations implied by $\Gamma_{k}$ define a $k$-dependent expectation value of the metric, a kind of mean field, $\left\langle g_{\mu \nu}\right\rangle_{k}$ :

$$
\frac{\delta \Gamma_{k}}{\delta g_{\mu \nu}(x)}\left[\langle g\rangle_{k}\right]=0 .
$$

At least when one applies the average action technique to Euclidean non-gauge theories on flat space, i.e. when the metric is non-dynamical, or to statistical mechanical systems on a rigid lattice, the action $\Gamma_{k}$ has the following properties [22]: (i) It defines an effective field theory at the momentum scale $k$. This means that every physical process which involves only a single momentum scale, say $p$, is well described by a tree level evaluation of $\Gamma_{k}$ with $k$ chosen as $k=p$. (ii) At least heuristically [22], $\Gamma_{k}$ may be interpreted as arising from a continuum version of a Kadanoff-Wilson block spin procedure, i.e. it defines the dynamics of "coarse grained" dynamical variables which are averaged over a certain region of Euclidean spacetime. Denoting the typical linear extension of the averaging region by $\ell$, one has $\ell \approx \pi / k$ in flat spacetime (at least for non-gauge theories). In this sense, $\Gamma_{k}$ can 
be thought of as a "microscope" with an adjustable resolving power $\ell=\ell(k)$.

In quantum gravity where the metric is dynamical it is not clear a priori to what extent (i) and (ii) continue to be valid. For sure the relationship between the IR cutoff $k$ and the "averaging scale" $\ell$ is more complicated in general. It is one of the purposes of the present paper to give a concrete definition of an "averaging" or "coarse graining" scale $\ell$, and to understand how it depends on $k$. Using this definition, along with certain qualitative properties of the RG trajectories of QEG, we shall demonstrate that the theory generates a minimal length scale in a dynamical way. The interpretation of this scale is rather subtle, however. One has to carefully distinguish different physical questions one could ask, because depending on the question a minimal length will, or will not become visible.

Let us assume we have solved the exact RG equation and picked a specific RG trajectory, a curve $k \mapsto \Gamma_{k}$ on theory space. Then we can write down the effective Einstein equations (11) along this trajectory and, after fixing appropriate symmetry and boundary conditions, find the corresponding family of mean field metrics $\left\{\left\langle g_{\mu \nu}\right\rangle_{k} ; 0 \leq k<\infty\right\}$. As for the interpretation of the average action approach in QEG, it is crucial to appreciate the fact that the infinitely many equations in (11), one at each scale $k$, are valid simultaneously, and that all the mean fields $\left\langle g_{\mu \nu}\right\rangle_{k}$ refer to one and the same physical system, a "quantum spacetime" in the QEG sense. The mean fields $\left\langle g_{\mu \nu}\right\rangle_{k}$ describe the metric structure in dependence on the length scale on which the spacetime manifold is probed. An observer exploring the structure of spacetime using a "microscope" of resolution $\ell(k)$ will perceive the universe as a Riemannian manifold with the metric $\left\langle g_{\mu \nu}\right\rangle_{k}$. While $\left\langle g_{\mu \nu}\right\rangle_{k}$ is a smooth classical metric at every fixed $k$, the quantum spacetime can have fractal properties because on different scales different metrics apply. In this sense the metric structure on the quantum spacetime is given by an infinite set $\left\{\left\langle g_{\mu \nu}\right\rangle_{k} ; 0 \leq k<\infty\right\}$ of ordinary metrics.

Recently it has been shown [23] that in asymptotically safe theories of gravity, at sub-Planckian distances, spacetime is indeed a fractal whose spectral dimension 
24] equals 2. It is quite remarkable that a similar dynamical dimensional reduction from 4 macroscopic to 2 microscopic dimensions has also been observed in Monte Carlo simulations of causal dynamical triangulations [25, 26, 27]. ${ }^{1}$

In order to understand the relation between $\ell$ and the IR cutoff $k$ we must recall the essential steps in the construction of the average action 3]. The formal starting point is the path integral $\int \mathcal{D} \gamma_{\mu \nu} \exp (-S[\gamma])$ over all metrics $\gamma_{\mu \nu}$, gauge fixed by means of a background gauge fixing condition [29]. Even without an IR cutoff, upon introducing sources and performing the usual Legendre transform one is led to an effective action $\Gamma\left[g_{\mu \nu} ; \bar{g}_{\mu \nu}\right]$ which depends on two metrics, the expectation value of $\gamma_{\mu \nu}$, denoted $g_{\mu \nu}$, and the non-dynamical background field $\bar{g}_{\mu \nu}$. It is well-known [29] that the functional $\Gamma\left[g_{\mu \nu}\right] \equiv \Gamma\left[g_{\mu \nu} ; \bar{g}_{\mu \nu}=g_{\mu \nu}\right]$ obtained by equating the two metrics generates the 1PI Green's functions of the theory.

The IR cutoff is implemented by first expanding the shifted integration variable $h_{\mu \nu} \equiv \gamma_{\mu \nu}-\bar{g}_{\mu \nu}$ in terms of eigenmodes of $\bar{D}^{2}$, the covariant Laplacian formed with the background metric $\bar{g}_{\mu \nu}$, and interpreting $\mathcal{D} h_{\mu \nu}$ as an integration over all expansion coefficients. Then a suppression term is introduced which damps the contribution of all $\bar{D}^{2}$-modes with eigenvalues smaller than $k^{2}$. Following the usual steps [22, 30] this leads to the scale dependent functional $\Gamma_{k}\left[g_{\mu \nu} ; \bar{g}_{\mu \nu}\right]$, and again the action with one argument is obtained by equating the two metrics: $\Gamma_{k}\left[g_{\mu \nu}\right] \equiv$ $\Gamma_{k}\left[g_{\mu \nu} ; \bar{g}_{\mu \nu}=g_{\mu \nu}\right]$. It is this action which appears in (11). Because of the identification of the two metrics it is, in a sense, the eigenmodes of $D^{2}$, constructed from the argument of $\Gamma_{k}[g]$, which are cut off at $k^{2}$. Note that neither the $g_{\mu \nu^{-}}$nor the $\bar{g}_{\mu \nu^{-}}$ argument of $\Gamma_{k}$ has any dependence on $k$. Therefore $\gamma_{\mu \nu}$ is expanded in terms of eigenfunctions of a fixed operator $\bar{D}^{2}$. Since its eigenfunctions are complete, we really integrate over all metrics when we lower $k$ from infinity to zero.

Note that a $k$-dependent mean field arises only at the point where we go "on shell" with $g_{\mu \nu}=\bar{g}_{\mu \nu}$ : the solution $\left\langle g_{\mu \nu}\right\rangle_{k}$ to eq. (11) depends on $k$, simply because $\Gamma_{k}$ does so.

\footnotetext{
${ }^{1}$ See also [28] for related discussions of fractal spacetimes within different theories of gravity.
} 
In ref. 23] an algorithm was proposed which allows the reconstruction of the "averaging" scale $\ell$ from the cutoff $k$. The input data is the set of metrics characterizing a quantum manifold, $\left\{\left\langle g_{\mu \nu}\right\rangle_{k}\right\}$. The idea is to deduce the relation $\ell=\ell(k)$ from the spectral properties of the scale dependent Laplacian $\boldsymbol{\Delta}_{k} \equiv D^{2}\left(\left\langle g_{\mu \nu}\right\rangle_{k}\right)$ built with the solution of the effective field equation. More precisely, for every fixed value of $k$, one solves the eigenvalue problem of $-\boldsymbol{\Delta}_{k}$ and studies in particular the properties of the eigenfunctions whose eigenvalue is $k^{2}$, or nearest to $k^{2}$ in the case of a discrete spectrum. We shall refer to an eigenmode of $-\boldsymbol{\Delta}_{k}$ whose eigenvalue is (approximately) the square of the cutoff $k$ as a "cutoff mode" (COM) and denote the set of all COMs by $\operatorname{COM}(k)$.

If we ignore the $k$-dependence of $\boldsymbol{\Delta}_{k}$ for a moment (as it would be appropriate for matter theories in flat space) the COMs are, for a sharp cutoff, precisely the last modes integrated out when lowering the cutoff, since the suppression term in the path integral cuts out all $h_{\mu \nu}$-modes with eigenvalue smaller than $k^{2}$.

For a non-gauge theory in flat space the coarse graining or averaging of fields is a well defined procedure, based upon ordinary Fourier analysis, and one finds that in this case the length $\ell$ is essentially the wave length of the last modes integrated out, the COMs.

This observation motivates the following tentative definition of $\ell$ in quantum gravity. We determine the COMs of $-\boldsymbol{\Delta}_{k}$, analyze how fast these eigenfunctions vary on spacetime, and read off a typical coordinate distance $\Delta x^{\mu}$ characterizing the scale on which they vary. For an oscillatory COM, for example, $\Delta x$ would correspond to an oscillation period. (In general there is a certain freedom in the precise identification of the $\Delta x^{\mu}$ belonging to a specific cutoff mode. This ambiguity can be resolved by refining the definition of $\Delta x^{\mu}$ on a case-by-case basis only. Since in the present paper only oscillatory eigenfunctions will be encountered we shall not be more specific here.) Finally we use the metric $\left\langle g_{\mu \nu}\right\rangle_{k}$ itself in order to convert $\Delta x^{\mu}$ to a proper length. This proper length, by definition, is $\ell$. Repeating the above steps for all values of $k$, we end up with a function $\ell=\ell(k)$. In general one will find 
that $\ell$ depends on the position on the manifold as well as on the direction of $\Delta x^{\mu}$. Since this position and direction dependence plays no role in the present paper we shall not indicate it notationally.

In the following $\ell$ will always denote the intrinsic length scale of the COMs obtained from the above algorithm. Our experience with theories in flat spacetime suggests that the COM scale $\ell$ is a plausible candidate for a physically sensible resolution function $\ell=\ell(k)$, but there might also be others, depending on the experimental setup one has in mind. Interestingly enough, we shall see that in quantum gravity the COM scale $\ell(k)$ has properties which are quite different from classical physics.

For later comparison with QEG it is instructive to go through the various steps of the algorithm in the standard case. We consider a classical flat Euclidean space with metric $\delta_{i j}$ and Laplacian $\boldsymbol{\Delta}=\sum \partial_{i}^{2}$. The eigenmodes of $-\boldsymbol{\Delta}$ with eigenvalue $k^{2}$ are $\sin (\vec{q} \cdot \vec{x})$ and $\cos (\vec{q} \cdot \vec{x})$ with $\vec{q}^{2}=k^{2}$. They vary between their maximal value +1 and their minimal value -1 in a coordinate interval $\Delta \vec{x}$ with $(\Delta \vec{x})^{2}=\pi^{2} / k^{2}$. The length corresponding to this coordinate distance, obtained with the metric $\delta_{i j}$, is

$$
\ell(k)=\sqrt{\delta_{i j} \Delta x^{i} \Delta x^{j}}=\pi / k .
$$

Thus we recover the expected inverse proportionality $\ell \propto 1 / k$.

The most difficult step in the construction of QEG spacetimes consists in finding the RG trajectories. The running action $\Gamma_{k}\left[g_{\mu \nu}\right]$ satisfies an exact functional RG equation [3]. In practice it is usually solved on a truncated theory space. In the Einstein-Hilbert truncation, for instance, $\Gamma_{k}$ is approximated by a functional of the form

$$
\Gamma_{k}[g]=(16 \pi G(k))^{-1} \int d^{4} x \sqrt{g}\{-R(g)+2 \Lambda(k)\}
$$

involving a running Newton constant $G(k)$ and cosmological constant $\Lambda(k)$.

For each $k$, the action (3) implies an effective field equation

$$
R_{\mu \nu}\left(\langle g\rangle_{k}\right)=\Lambda(k)\left\langle g_{\mu \nu}\right\rangle_{k}
$$




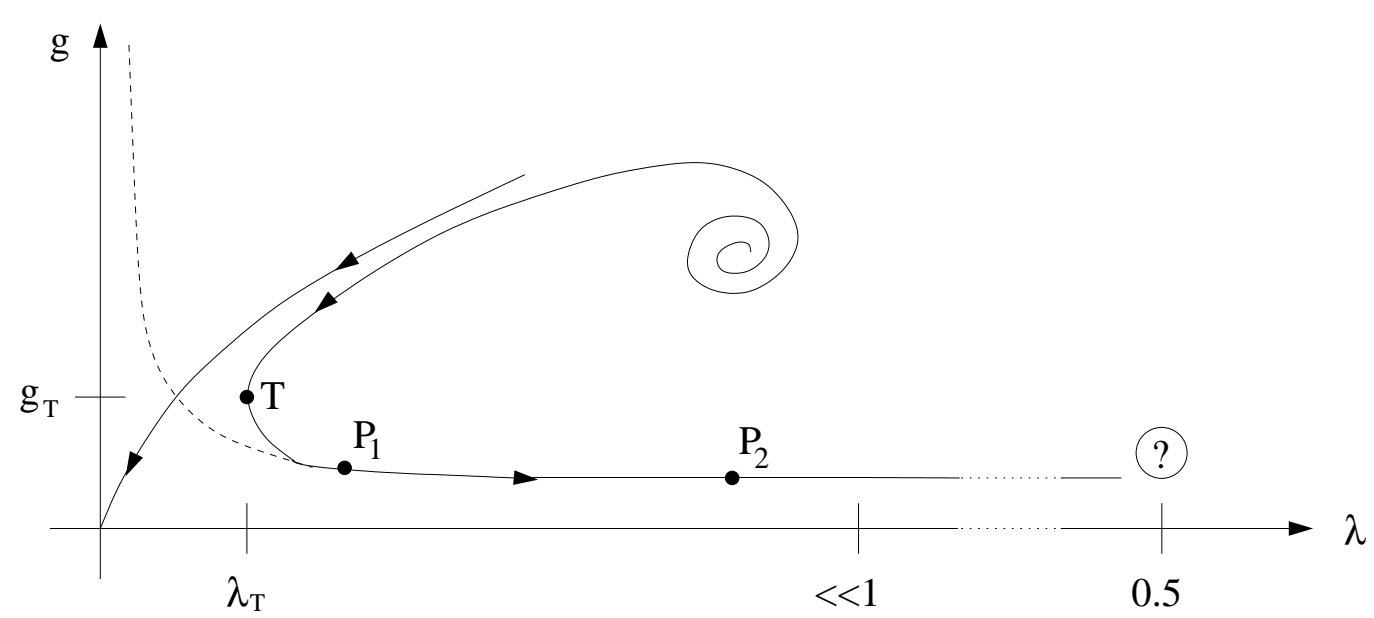

Figure 1: A Type IIIa trajectory and the separatrix. The dashed line is a trajectory of the canonical RG flow. The arrows point in the direction of decreasing $k$.

Note that the running Newton constant $G(k)$ does not appear in this effective Einstein equation. It enters only when matter fields are introduced.

The qualitative properties of the trajectories following from the Einstein-Hilbert approximation are well-known by now [6]. Fig. 1 shows a "Type IIIa" trajectory which would be the type that is presumably realized in the real universe since it is the only type that has a positive Newton's constant $G(k)$ and a small positive cosmological constant $\Lambda(k)$ at macroscopic scales. In Fig. 1 it is plotted in terms of the dimensionless parameters $g(k) \equiv k^{2} G(k)$ and $\lambda(k) \equiv \Lambda(k) / k^{2}$ and compared to the canonical trajectory of classical gravity (dashed curve) with $\Lambda=$ const and $G=$ const. The Type IIIa trajectory contains the following four parts, with increasing values of the cutoff $k$ :

i) The classical regime for small $k$ where the trajectory is identical to the canonical one. (In Fig. 1 the segment between the points $P_{1}$ and $P_{2}$.)

ii) The turnover regime where the trajectory, close to the Gaussian fixed point at $g=\lambda=0$, begins to depart from the canonical one and turns over to the separatrix which connects the Gaussian with the non-Gaussian fixed point $\left(g_{*}, \lambda_{*}\right)$. By defini- 
tion, the coordinates of the turning point $T$ are $g_{T}$ and $\lambda_{T}$, and it is passed at the scale $k=k_{T}$.

iii) The growing $\Lambda$ regime where $G(k)$ is approximately constant but $\Lambda(k)$ runs proportional to $k^{4}$.

iv) The fixed point regime where the trajectory approaches the non-Gaussian fixed point in an oscillating manner. Directly at the fixed point one has $g(k) \equiv g_{*}$ and $\lambda(k) \equiv \lambda_{*}$, and therefore $G(k) \propto k^{-2}$ and $\Lambda(k) \propto k^{2}$ for $k \rightarrow \infty$. The non-Gaussian fixed point is responsible for the nonperturbative renormalizability of the theory.

The behavior of the trajectory in the extreme infrared is not yet known since the Einstein-Hilbert approximation breaks down when $\lambda(k)$ approaches the value $1 / 2$. A more general truncation is needed to approximate the $R G$ trajectory in that region. For this reason the classical region i) does not necessarily extend to $k=0$, and we speak about "laboratory" scales for values of $k \equiv k_{\text {lab }}$ in the region where $G$ and $\Lambda$ are constant. The Planck mass is then defined as

$$
m_{\mathrm{Pl}} \equiv 1 / \sqrt{G\left(k_{\mathrm{lab}}\right)}
$$

In the regimes i), ii) and iii) the trajectory is well approximated by linearizing the RG flow about the Gaussian fixed point. In terms of the dimensionful parameters one finds that in its linear regime $G(k)=$ const and 31

$$
\Lambda(k)=\Lambda_{0}\left[1+\left(k / k_{T}\right)^{4}\right]
$$

where $\Lambda_{0}$ is a constant. The corresponding dimensionless $\lambda=\Lambda / k^{2}$ runs according to

$$
\lambda(k)=\Lambda_{0}\left[\left(\frac{1}{k}\right)^{2}+\left(\frac{k}{k_{T}^{2}}\right)^{2}\right]
$$

Note that this function is invariant under the "duality transformation" $k \mapsto k_{T}^{2} / k$ :

$$
\lambda(k)=\lambda\left(k_{T}^{2} / k\right)
$$


For further details and a discussion of the other types of trajectories we refer to [6] and [31]. The analysis in the following sections of this paper refers entirely to trajectories of Type IIIa.

The rest of this paper is organized as follows. After discussing the concept of scale dependent distances in Section 2 we introduce, in Section 3, a specific model of a fractal spacetime, the four-sphere in the sense of QEG. On this spacetime all the constructions reviewed in the Introduction can be performed explicitly. In particular, in Section 4, we determine the corresponding cutoff modes, the set $\operatorname{COM}(k)$, and derive their proper coarse graining scale $\ell$. While $\ell$ can become arbitrarily small we shall demonstrate in Section 5 that the angular resolution of the COMs cannot be increased beyond a certain point, rendering the $S^{4}$ of QEG a kind of "fuzzy sphere". In Section 6 we analyze this fuzzyness from the point of view of a macroscopic observer. Then, in Section 7, we discuss the idea of an "intrinsic" distance of two spacetime points, as well as a new kind of duality transformations exchanging large and small distances. Section 8 contains a brief summary of the results.

\section{Scale dependent distances}

With a fixed classical metric, the (geodesic, say) distance of two given points $x$ and $y$ on a Riemannian manifold reads

$$
L(x, y) \equiv \int_{\mathcal{C}_{x y}}\left(g_{\mu \nu} d x^{\mu} d x^{\nu}\right)^{1 / 2}
$$

where $\mathcal{C}_{x y}$ denotes the geodesic connecting $x$ to $y$. (For simplicity, we assume that $x$ and $y$ are close enough so that there are no caustics.) In a quantum spacetime, $g_{\mu \nu}$ is replaced by the set of metrics $\left\{\left\langle g_{\mu \nu}\right\rangle_{k}\right\}$. As a result, the distance from $x$ to $y$ depends on $k$ now:

$$
L_{k}(x, y) \equiv \int_{\mathcal{C}_{x y}}\left(\left\langle g_{\mu \nu}\right\rangle_{k} d x^{\mu} d x^{\nu}\right)^{1 / 2} .
$$

The interpretation of this $k$-dependent distance is as follows. If $k$ parametrizes the "resolution of the microscope" with which the spacetime is observed, the metric $\left\langle g_{\mu \nu}\right\rangle_{k}$ and correspondingly the distance $L_{k}(x, y)$ pertain to a specific scale of reso- 
lution, and different observers, using microscopes of different $k$-values, will measure different lengths in general. This $k$-dependence of lengths is analogous to the "coastline of England phenomenon" well known from fractal geometry [32, 24]. What complicates the situation in QEG is that $k$ has no direct physical meaning a priori and can be related to an averaging scale only indirectly, for instance via the properties of the COMs.

\section{The QEG four-sphere}

The QEG four-sphere is a manifold in the QEG sense, i.e. supplied with a family of infinitely many metrics $\left\{\left\langle g_{\mu \nu}\right\rangle_{k} \mid k=0, \cdots, \infty\right\}$. To be specific, it is the family of maximally symmetric solutions of (4) with positive curvature. It exists only provided $\Lambda(k)>0$, which is the case for all type IIIa trajectories. Strictly speaking, as long as we are restricted to the Einstein-Hilbert truncation, the family of metrics starts not at $k=0$, but at some $k=k_{\min }$, beyond which (towards the infrared) the trajectory cannot be continued.

We may parametrize the four-sphere by coordinates $(\zeta, \eta, \theta, \phi)$ with ranges $0<$ $\zeta, \eta, \theta<\pi$ and $0 \leq \phi<2 \pi$. The metric can be written as

$$
\left\langle g_{\mu \nu}\right\rangle_{k} d x^{\mu} d x^{\nu}=r^{2}(k)\left[d \zeta^{2}+\sin ^{2} \zeta\left(d \eta^{2}+\sin ^{2} \eta\left(d \theta^{2}+\sin ^{2} \theta d \phi^{2}\right)\right)\right],
$$

where $r(k)$ is the $k$-dependent radius of the $S^{4}$. To determine it, one has to insert the ansatz (11) into the effective vacuum field equation (4). The result is

$$
r(k)=\sqrt{3 / \Lambda(k)}
$$

The family of metrics (11), (12) constitutes a concrete example of a quantum spacetime as it was discussed in ref. [23]. In particular it has a scale dependent, in general non-integer fractal dimension. Both the spectral dimension $\mathcal{D}_{S}$ and the one based upon the anomalous dimension $\eta_{N}$ interpolate between 4 at macroscopic distances and 2 on microscopic scales. Contrary to a Brownian curve or the coastline of England the fractal dimension of the quantum spacetime is equal or smaller than the classical one. The reason is that, according to (11) and (12), distances decrease 
when we increase the cutoff $k$. The metric scales as $\left\langle g_{\mu \nu}\right\rangle_{k} \propto 1 / \Lambda(k)$ so that in the fixed point regime $\left\langle g_{\mu \nu}\right\rangle_{k} \propto 1 / k^{2}$ implying $L_{k}(x, y) \propto 1 / k$ for any (geodesic) distance.

In fact, on the sphere it is easy to write down the geodesic distance (10) explicitly. Without loss of generality we may assume that the two points $x$ and $y$ are both located on the equator $\zeta=\eta=\theta=\pi / 2$. Denoting their $\phi$-angles by $\phi(x)$ and $\phi(y)$, respectively, and exploiting that on the equator

$$
\left\langle g_{\phi \phi}\right\rangle_{k}=r^{2}(k)=3 / \Lambda(k)
$$

the geodesic distance $L_{k}(x, y)=\sqrt{\left\langle g_{\phi \phi}\right\rangle} \int d \phi=r(k) \int d \phi$ reads

$$
\begin{aligned}
L_{k}(x, y) & =\sqrt{3 / \Lambda(k)}|\phi(x)-\phi(y)| \\
& =\frac{1}{k} \sqrt{\frac{3}{\lambda(k)}}|\phi(x)-\phi(y)|
\end{aligned}
$$

We shall come back to this expression later on.

\section{The cutoff modes and their resolving power}

On the quantum $S^{4}$, the scalar eigenfunctions of $-\boldsymbol{\Delta}_{k}$ have the discrete eigenvalues

$$
\mathcal{E}_{n}=\frac{n(n+3)}{r^{2}(k)}, \quad n=0,1,2,3, \cdots
$$

with degeneracies

$$
D_{n}=\frac{1}{6}(n+1)(n+2)(2 n+3) .
$$

The eigenmodes are labeled by four integer quantum numbers $n, l_{1}, l_{2}$ and $m$, where $n \geq l_{1} \geq l_{2} \geq|m|$. They have the form

$$
Y_{n l_{1} l_{2} m}(\zeta, \eta, \theta, \phi)={ }_{4} \bar{P}_{n}^{l_{1}}(\zeta){ }_{3} \bar{P}_{l_{1}}^{l_{2}}(\eta){ }_{2} \bar{P}_{l_{2}}^{m}(\theta) \frac{1}{\sqrt{2 \pi}} e^{i m \phi}
$$

Here ${ }_{i} \bar{P}_{k}^{j}(\alpha)$ are generalized associated Legendre functions, for details see e.g. ref. 33.

Let us determine the associated set of cutoff modes $\operatorname{COM}(k)$, i.e. the eigenfunctions with $-\boldsymbol{\Delta}_{k}$-eigenvalue as close as possible to $k^{2}$. Inserting $\mathcal{E} \approx k^{2}$ into (15) and 
using eq. (12) for $r(k)$, we find the following equation for the $n$-quantum number of the COMs at scale $k$ :

$$
n(k)(n(k)+3) \approx \frac{3 k^{2}}{\Lambda(k)}=\frac{3}{\lambda(k)} .
$$

The eigenvalues for the vector and tensor modes are slightly different, but for large $n$ the difference becomes negligible and the spectrum is to a good approximation continuous. We will use this continuum approximation since we are interested in small angular distances $\Delta \phi$ anyway. Then eq.(18) becomes

$$
n(k) \approx \sqrt{\frac{3}{\lambda(k)}} .
$$

Obviously $n(k)$ is indeed large if $\lambda(k) \ll 1$.

Since the eigenvalue belonging to $Y_{n l_{1} l_{2} m}$ depends on $n$ only, the set $\operatorname{COM}(k)$ consists of all harmonics $Y_{n l_{1} l_{2} m}$ with $n$ fixed by eq. (19) and the other quantum numbers $l_{1}, l_{2}$ and $m$ arbitrary except for the constraint $n \geq l_{1} \geq l_{2} \geq|m|$. If $\lambda(k) \ll 1$, the dimension of $\operatorname{COM}(k)$ as a vector space is approximately $n(k)^{3} / 3 \approx$ $\sqrt{3} \lambda(k)^{-3 / 2} \gg 1$.

Apart from its obvious dependence on the scale, the set $\operatorname{COM}(k)$ depends on the RG trajectory via the function $\lambda(k)$ which determines $n(k)$. It is important to note that the function $\lambda=\lambda(k)$ is not invertible in general and that different $k$ 's can lead to the same $\operatorname{COM}(k)$. Let us look at the Type IIIa trajectory in Fig. 1 as an example. First we concentrate on its part close to the turning point, staying away from the spiraling regime in the UV, and the IR region where the Einstein-Hilbert truncation breaks down. We observe then that for every scale $k<k_{T}$ below the turning point there exists a corresponding scale $k^{\sharp}>k_{T}$ which has the same $\lambda$-and therefore $n$-value. As a result, the corresponding cutoff modes are equal at the two scales: $\operatorname{COM}(k)=\operatorname{COM}\left(k^{\sharp}\right)$.

If the turning point is sufficiently close to the Gaussian fixed point, and $k$ is not too far from $k_{T}$, we may use the linearization (77) for an approximate determination of $k^{\sharp}$. Because of the "duality symmetry" (8) it is given by

$$
k^{\sharp}=k_{T}^{2} / k \text {. }
$$


Thus, in the linear regime, $\operatorname{COM}(k)=\operatorname{COM}\left(k_{T}^{2} / k\right)$.

Outside the linear regime the relation between $k^{\sharp}$ and $k$ is more implicated. The situation becomes even more involved in the regime close to the non-Gaussian fixed point. There, because of the spiral structure, very many different $k$-values have the same $\lambda(k)$ and $\operatorname{COM}(k)$.

Next we analyze the degree of position dependence of the functions in $\operatorname{COM}(k)$ and try to quantify their "resolving power". In order to convert the estimate for $n(k)$, eq. (19), to an angular resolution we note that it is sufficient to do so for one position and one direction. By the translation and rotation symmetries of the sphere, the resolution will be the same at any other point and in any other direction. We therefore choose to determine the angular resolution of the modes along the equator $\zeta=\eta=\theta=\pi / 2$.

Two of the $\boldsymbol{\Delta}_{k}$-eigenfunctions with eigenvalue $n(k)$, namely $Y_{ \pm} \equiv Y_{n n n \pm n}$, oscillate most rapidly as a function of $\phi$, and we shall use them in order to define the angular resolution. Their $\phi$-dependence is $e^{ \pm i n \phi}$ and the corresponding angular resolution is

$$
\Delta \phi(k)=\frac{\pi}{n(k)}=\pi \sqrt{\frac{\lambda(k)}{3}} .
$$

As expected, the angular resolution implied by the COMs depends on the RG trajectory. It does so only via the function $\lambda=\lambda(k)$ and, as a result, can be of the same size for different values of $k$. In particular $\Delta \phi(k)=\Delta \phi\left(k^{\sharp}\right)$ and, in the linear regime, $\Delta \phi(k)=\Delta \phi\left(k_{T} / k^{2}\right)$.

The angular separation (21) is the coordinate distance of two consecutive zeros of the real or imaginary part of $Y_{ \pm}$along the equator $\zeta=\eta=\theta=\pi / 2$. By definition, the COM scale $\ell$ is the proper length corresponding to $\Delta \phi(k)$ as computed with the metric $\left\langle g_{\mu \nu}\right\rangle_{k}$ of eqs. (11), (12). It is given by $\ell(k)=\Delta \phi(k) \sqrt{\left\langle g_{\phi \phi}\right\rangle_{k}}$ or, using eq. (13), $\ell(k)=\Delta \phi(k) \sqrt{3 / \Lambda(k)}=(1 / k) \Delta \phi(k) \sqrt{3 / \lambda(k)}$. Hence, with (21),

$$
\ell(k)=\frac{\pi}{k} \text {. }
$$

So we find that, as in theories on a classical flat spacetime, the natural proper length scale $\ell$ of the $\operatorname{COM}(k)$-modes is just $\pi / k$. Thanks to the symmetry of the sphere it 
is neither position nor direction dependent.

\section{Lower bound on the angular resolution}

Taking just the (perhaps expected) result $\ell \propto 1 / k$, it seems as if nothing remarkable had happened. But the surprising effects appear in our result for the angular resolution, eq. (21). As we can see from the flow diagram of Fig. 1, $\lambda(k)$ takes on a minimum value $\lambda_{T}$ at the turning point T. Only the Type IIIa trajectories have this turning point, and this is one of the features that makes them particularly interesting. In fact, as $\lambda(k) \geq \lambda_{T}$ for any scale $k$, we conclude that the angular resolution $\Delta \phi(k)$ is bounded below by the minimum angular resolution

$$
\Delta \phi_{\min }=\pi \sqrt{\frac{\lambda_{T}}{3}}
$$

Stated differently, there does not exist any cutoff $k$ for which $\Delta \phi(k)$ would be smaller than $\Delta \phi_{\min }$. On the other hand, angular resolutions between $\Delta \phi_{\min }$ and $\Delta \phi_{*}$, defined by

$$
\Delta \phi_{*} \equiv \pi \sqrt{\frac{\lambda_{*}}{3}},
$$

are realized for at least two scales $k$, one of them above, the other below $k_{T}$.

What has happened here? Coming from small $k$, we travel along the RG trajectory and follow its spherical solutions, observing spacetime with a "microscope" of variable proper resolution $\ell(k)$. At first, in the classical regime, an increase of $k$ leads to the resolution of finer and finer structures since $\Lambda=$ const implies $\Delta \phi(k) \propto 1 / k$. For the canonical RG trajectory, this behavior would continue even for $k \rightarrow \infty$. In quantum gravity, however, in region ii), the sphere starts to shrink, due to a growing cosmological constant $\Lambda(k)$. At the turning point scale $k_{T}$ at which $\lambda(k)$ assumes its minimum $\lambda_{T}$, the shrinking becomes faster than the improvement of the resolution $\left(r(k) \propto k^{-2}\right.$ in region iii)). Although we can resolve smaller and smaller proper distances, this is of no use, since the sphere is shrinking so fast that a smaller proper length corresponds to a larger angular distance. Finally, in the fixed point regime (at large angles although this is an ultraviolet fixed point!), the shrinking 
slows down to a rate that cancels exactly the improved resolution of the microscope $\left(r(k) \propto k^{-1}\right)$ so that the angular resolution approaches a constant value $\Delta \phi_{*}$ after the oscillations have been damped away. (Depending on the value of $\lambda_{*}$ it may even happen that the UV fixed point corresponds to so large angular scales that the approximation $n \gg 1$ breaks down.)

The minimum of $\Delta \phi$ at the turning point is equivalent to a maximum of the $n$ quantum number the COMs can have:

$$
n_{\max } \approx \sqrt{\frac{3}{\lambda_{T}}} .
$$

Does this mean that in the fundamental path integral underlying the flow equation not all quantum fluctuations are integrated out when $k$ is lowered from infinity to $k=0$ ? Are the modes with $n>n_{\max }$ left out? The answer to these questions is a clear no. One has to carefully distinguish the quantum metric $\gamma_{\mu \nu}$, the variable of integration in the path integral $\int \mathcal{D} \gamma \exp (-S[\gamma]+\ldots)$, from its expectation value, or mean field, $\left\langle g_{\mu \nu}\right\rangle_{k}$. As we explained above, the shifted functional integration over $h_{\mu \nu}=\gamma_{\mu \nu}-\bar{g}_{\mu \nu}$ which is implicit in the definition of $\Gamma_{k}\left[g_{\mu \nu}, \bar{g}_{\mu \nu}\right]$ is organized according to the eigenmodes of the covariant Laplacian $\bar{D}^{2}$ constructed from the arbitrary but fixed background metric $\bar{g}_{\mu \nu}$. This integration is performed before $g_{\mu \nu}$ and $\bar{g}_{\mu \nu}$ are identified and the result is equated to $\left\langle g_{\mu \nu}\right\rangle_{k}$. At scale $k$, the $-\bar{D}^{2}$ modes down to eigenvalues $\approx k^{2}$ are integrated out, and therefore on the way from $k=\infty$ to $k=0$ it is really all modes of $h_{\mu \nu}$ and therefore $\gamma_{\mu \nu}$ that are integrated out since the eigenfunctions of $\bar{D}^{2}$ form a complete system.

The results of the present paper should instead be thought of as reflecting properties of the mean field $\left\langle g_{\mu \nu}\right\rangle_{k}$. Rather than the spectrum of the $k$-independent operator $\bar{D}^{2}$ we analyzed that of the explicitly $k$-dependent Laplacian $D^{2}\left(\left\langle g_{\mu \nu}\right\rangle_{k}\right)$; its explicit $k$-dependence is due to the scale dependence of the on-shell metric, the solution to the effective Einstein equation. Our argument reveals that the effective spacetime with the running on-shell metric cannot support harmonic modes of arbitrarily fine angular resolution.

This phenomenon is a purely dynamical one; the finite resolution is not built 
in at the kinematical (i.e. $\gamma_{\mu \nu^{-}}$) level, as it would be the case, for instance, if the fundamental theory was defined on a lattice. It is also important to stress that, if the non-Gaussian fixed point exists, the Green's functions $G_{n}\left(x_{1}, x_{2}, \cdots, x_{n}\right)$ can be made well defined at all non-coincident points, i.e. for arbitrarily small coordinate distances among the $x_{i}^{\mu}$ 's. Those Green's functions contain information even about angular scales smaller than $\Delta \phi_{\min }$, in particular they "know" about the asymptotic safety of the theory which manifests itself only at scales $k \gg k_{T}$.

In fact, the argument leading to the finite resolution $\Delta \phi_{\min }$ is fairly independent of the high energy behavior of the theory. The crucial ingredient in the above reasoning was the occurrence of a minimum value for $\lambda(k)$. This minimum occurs as a direct consequence of the $k^{4}$-running of $\Lambda(k)$ given in eq. (6); how the trajectory continues beyond the scaling regime of the Gaussian fixed point was not important. However, this $k^{4}$-running occurs already in standard perturbation theory, simply reflecting the quartic divergences of all vacuum diagrams. From this point of view our argument is rather robust; it would apply even to a non-asymptotically safe theory in which the impact of the perturbative $k^{4}$-running is taken seriously.

Moreover, while the above argument was formulated within the Einstein-Hilbert truncation, it is likely to be still valid in more general truncations and the exact theory. The only prerequisite is that, near $k=k_{T}$, the effective field equations must admit $S^{4}$ solutions. For some of the trajectories this will always be the case presumably.

The upper bound on the angular momentum like quantum number $n$ is reminiscent of the "fuzzy sphere" constructed in ref. [34. While in the case of the fuzzy sphere the finite angular resolution is put in "by hand", in the present case it emerges as a consequence of the quantum gravitational dynamics .

\section{Minimum proper length for a macroscopic observer}

A macroscopic, classical observer would find it natural to employ the metric

$$
g_{\mu \nu}^{\text {macro }}=\left\langle g_{\mu \nu}\right\rangle_{k_{\text {lab }}},
$$


where $k_{\text {lab }}$ is any scale in the classical regime in which $G$ and $\Lambda$ do not run (in Fig. 1 between the points $P_{1}$ and $P_{2}$ ). Using this metric we can define a "macroscopic distance" for any two given points $x$ and $y$ :

$$
L^{\text {macro }}(x, y) \equiv \int_{\mathcal{C}_{x y}}\left(g_{\mu \nu}^{\text {macro }} d x^{\mu} d x^{\nu}\right)^{1 / 2} .
$$

It is instructive to ask which proper length the macroscopic observer using $g_{\mu \nu}^{\text {macro }}$ would ascribe to the finite coordinate distance $\Delta \phi_{\min }$. Denoting this proper length by $L_{\min }^{\text {macro }}$ we obtain

$$
L_{\min }^{\operatorname{macro}}=r\left(k_{\mathrm{lab}}\right) \Delta \phi_{\min }=\left[3 / \Lambda\left(k_{\mathrm{lab}}\right)\right]^{1 / 2} \Delta \phi_{\min },
$$

and with eq. (23),

$$
L_{\min }^{\operatorname{macro}}=\frac{\pi}{k_{T}} \sqrt{\frac{\Lambda\left(k_{T}\right)}{\Lambda\left(k_{\mathrm{lab}}\right)}}=\frac{\pi}{k_{T}}\left[\frac{2}{1+\left(k_{\mathrm{lab}} / k_{T}\right)^{4}}\right]^{1 / 2} .
$$

In the second equality of (29) we assumed that the turning point is sufficiently close to the Gaussian fixed point so that eq. (6) is a good approximation for $\Lambda(k)$. (For the trajectory which seems realized in Nature this is indeed the case.) If $k_{\text {lab }} \ll k_{T}$ we find that $L_{\min }^{\text {macro }}$ is essentially the same as the turning point scale:

$$
L_{\min }^{\operatorname{macro}}=\sqrt{2} \pi k_{T}^{-1}
$$

Remarkably, this minimal proper length is different in general from the Planck length which is usually thought to set the minimal length scale. Using the linearized flow one can show [31] that $k_{T} / m_{\mathrm{Pl}} \approx \sqrt{g_{T}}$. So, if $g_{T}$ is small, $L_{\text {min }}^{\text {macro }}$ can be much larger than $\ell_{\mathrm{Pl}} \equiv m_{\mathrm{Pl}}^{-1}$. The trajectory realized in Nature seems to be an extreme example: It has $g_{T} \approx 10^{-60}$, implying $k_{T}^{-1} \approx 10^{30} \ell_{\mathrm{Pl}} \approx 10^{-3} \mathrm{~cm}$, and $L_{\text {min }}^{\text {macro is of the }}$ same order of magnitude.

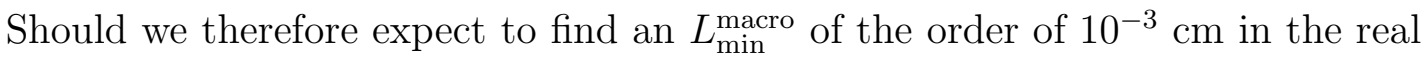
world? The answer is no, most probably. The reason is that our present discussion is based upon the vacuum field equations where it is the value of the cosmological constant alone which determines the curvature of spacetime. In presence of matter, 
the scale dependence of $\Lambda$ can have an observable effect only if the vacuum energy density $\rho_{\Lambda} \equiv \Lambda / 8 \pi G$ is comparable to the matter energy density (including the matter energy density of the measuring device).

\section{Intrinsic distance and scale doubling}

In fractal geometry and any framework involving a length scale dependent metric one can try to define an "intrinsic" distance of any two points $x$ and $y$ by adjusting the resolving power of the "microscope" in such a way that the length scale it resolves equals approximately the, yet to be determined, intrinsic (geodesic) distance from $x$ to $y .^{2}$

To be concrete, let us fix two points $x$ and $y$ and let us try to assign to them a cutoff scale $k \equiv k(x, y)$ which satisfies

$$
L_{k(x, y)}(x, y)=\ell(k(x, y))
$$

Eq. (31) is a self-consistency condition for $k(x, y)$ : the LHS of (31) is the distance from $x$ to $y$ as seen by a microscope with $k=k(x, y)$, and the RHS is precisely the resolution of this microscope. If (31) has a unique solution $k(x, y)$ one defines the intrinsic distance by setting

$$
L_{\text {in }}(x, y) \equiv L_{k(x, y)}(x, y)
$$

Since $\ell(k)=\pi / k$, this distance is essentially the inverse cutoff scale:

$$
L_{\mathrm{in}}(x, y)=\frac{\pi}{k(x, y)}
$$

Let us evaluate the self-consistency condition (31). Without loss of generality we may assume again that $x$ and $y$ are located on the equator of $S^{4}$ so that eq. (14) applies. Then, by virtue of (22), eq. (31) boils down to the following implicit equation for $k(x, y)$ :

$$
\lambda(k(x, y))=\frac{3}{\pi^{2}}|\phi(x)-\phi(y)|^{2} .
$$

\footnotetext{
${ }^{2}$ This kind of dynamical adjustment of the resolution has also been used in the RG improvement of black hole [35] and cosmological [36]-42] spacetimes, see in particular ref. [4].
} 
Recalling the properties of the function $\lambda(k)$ for a Type IIIa trajectory we see that (34) does not admit a unique solution for $k(x, y)$. If $x$ and $y$ are such that $|\phi(x)-\phi(y)|<\Delta \phi_{\min }$ it possesses no solution at all, and if $|\phi(x)-\phi(y)|>\Delta \phi_{\min }$ it has at least two solutions. Staying away from the deep UV and IR regimes, every solution $k(x, y)<k_{T}$ on the lower branch of the RG trajectory has a partner solution $k(x, y)^{\sharp}>k_{T}$ on its upper branch. As a result, the intrinsic distance of $x$ and $y$ is either undefined, or there exist at least two different lengths which satisfy the self-consistency condition (31).

In the linear regime where $k^{\sharp}=k_{T}^{2} / k$, the two lengths $L_{\text {in }}(x, y)=\pi / k(x, y)$ and $L_{\text {in }}(x, y)^{\sharp}=\pi / k(x, y)^{\sharp}$ are related by

$$
L_{\text {in }}(x, y)^{\sharp}=\frac{L_{T}^{2}}{L_{\text {in }}(x, y)}
$$

where $L_{T} \equiv \pi / k_{T}$. Eq. (35) is reminiscent of the dualities in string theory. If $L_{\text {in }}(x, y)$ is large compared to the turning point length scale $L_{T}$, the "dual" scale $L_{\text {in }}(x, y)^{\sharp}$ is small. In the extreme case, when applied to Nature's RG trajectory, the duality (35) would even exchange the Planck- with the Hubble-regime: $L_{\text {in }}(x, y) \approx H_{0}^{-1}$ implies $L_{\text {in }}(x, y)^{\sharp} \approx l_{\mathrm{Pl}}$.

Thus we found one more instance where a quite unexpected "doubling" of $k$ scales makes its appearance. The first phenomenon of this kind which we encountered was the exact equality of $\operatorname{COM}(k)$ with $\operatorname{COM}\left(k^{\sharp}\right)$, and later on we saw that also the angular resolution $\Delta \phi$ is exactly the same at $k$ and $k^{\sharp}$. We shall refer to this phenomenon as a "scale doubling", keeping in mind however that in the spiraling regime there are many more $k$-values with the same $\operatorname{COM}(k)$ and $\Delta \phi(k)$.

At a formal level the origin of the doubling is easy to understand. It is due to the "back bending" of the RG trajectory at the turning point $T$ which implies that the function $\lambda=\lambda(k)$ assumes a minimum at a finite scale $k=k_{T}$. Only the trajectories of Type IIIa possess a turning point of this kind, and this is one of the reasons why they are particularly interesting and we restricted our discussion to them. It is important to understand that the occurrence of this turning point is a pure quantum effect. Switching off the renormalization (i.e. quantum) effects, 
the Type IIIa trajectory is substituted by the canonical trajectory shown in Fig. 1 which has no turning point and no scale doubling therefore.

While its origin is quite clear, the physical implications of the scale doubling and the duality symmetry are somewhat mysterious. To some extent the difficulty of giving a precise physical meaning to them is related to the fact that one actually should define the "resolution of the microscope" in terms of realistic experiments rather than the perhaps too strongly idealized mathematical model of a measurement based upon the COMs. For various reasons it seems premature to assign a direct observational relevance to the minimal angular resolution and the scale doubling:

(i) We found that only the coordinate distance $\Delta \phi(k)$ assumes a minimum, but not the corresponding proper distance computed with the running metric $\left\langle g_{\mu \nu}\right\rangle_{k}$. In particular the resolution function $\ell(k)=\pi / k$ is exactly the same as in flat space. (But nevertheless the COM-microscope is unable to distinguish points with an angular separation below $\Delta \phi_{\min }$ !)

(ii) Our analysis applies to pure gravity. In presence of matter the "fuzziness" of the $S^{4}$ can become visible probably only at scales where the cosmological constant dominates the energy density.

(iii) As a special case of (ii), the fuzziness might be masked by the backreaction of a realistic (i.e. gravitating) measuring apparatus on the spacetime structure.

(iv) As for a possible physical significance of the duality symmetry it is to be noted that the two scales which it relates, $k<k_{T}$ and $k^{\sharp}>k_{T}$, have a rather different status as far as quantum fluctuations about the mean field metric $\left\langle g_{\mu \nu}\right\rangle_{k}$ are concerned. The structure of the exact RG equation is such that the fluctuations are the larger the stronger the renormalization effects are. As a result, the metric fluctuations about $\left\langle g_{\mu \nu}\right\rangle_{k^{\sharp}}$ on the upper branch are certainly larger than at the dual point on the lower branch of the RG trajectory. 
Clearly more work is needed in order to understand these issues better. We emphasize, however, that the above discussion deals only with the interpretation of QEG, and not with its basic construction along the lines of the asymptotic safety scenario. As we pointed out already, the existence of a finite $\Delta \phi_{\min }$ is perfectly consistent with having integrated out all modes of the quantum metric. Throughout the present paper we assumed that the quantum theory has been constructed already and that its RG trajectories are known. What we did is to derive the running mean field metric for a given trajectory and to analyze its properties, in particular with respect to the running set of cutoff modes $\operatorname{COM}(k)$ it gives rise to.

\section{Summary}

In this paper we constructed a special example of a "QEG spacetime", the quantum 4-sphere, and analyzed its properties. We were particularly interested in the relationship between the IR cutoff $k$ and the coarse graining scale $\ell$. Strictly speaking this scale, the resolving power of the "microscope" with which we observe the spacetime structure, depends in a complicated way on how this "microscope" is realized in practice, i.e. on what kind of experiment we perform in order to probe spacetime. Rather than analyzing possible experiments explicitly we used a simple and natural mathematical model for the resolving power, namely we defined $\ell$ to be the typical proper length scale of the cutoff modes. The main motivations for this definition are that on a classical spacetime it reproduces the standard result, and that it is an intrinsic property of any RG trajectory: given the trajectory $k \mapsto \Gamma_{k}$, we can solve for $\left\langle g_{\mu \nu}\right\rangle_{k}$ at each scale, build $\boldsymbol{\Delta}_{k}$ from it, and solve its eigenvalue problem to find $\operatorname{COM}(k)$.

Applying this algorithm to the quantum $S^{4}$ we found that the IR cutoff and the proper coarse graining scale are related in exactly the same way as in classical flat space: $\ell(k)=\pi / k$. In obtaining this result it was crucial to employ the running metric $\left\langle g_{\mu \nu}\right\rangle_{k}$ for converting coordinate to proper distances.

Regarding the question as to whether QEG generates a minimal length dynam- 
ically, again, one should in principle analyze realistic experiments on a case-by-case basis. As a first step in this direction we analyzed this issue within the COM-model of the "microscope". The results are:

(a) There is no lower bound on proper distances measured with the running metric $\left\langle g_{\mu \nu}\right\rangle_{k}$.

(b) There is a nonzero minimal angular separation $\Delta \phi_{\min }$, i.e. a minimal coordinate distance that the COMs can resolve.

(c) There is a nonzero lower bound $L_{\text {min }}^{\text {macro }}$ on proper distances measured with a fixed macroscopic metric.

The statements (a) and (b) above can be true simultaneously only thanks to the running of the gravitational parameters: Increasing $k$ the on-shell spacetime shrinks, and this effect counteracts our ability to separate two points by making $k$ larger. This is the physical origin of the finite angular resolution $\Delta \phi_{\min }$. Converting $\Delta \phi_{\min }$ to a proper length with the running metric $\left\langle g_{\mu \nu}\right\rangle_{k}$, the resulting length becomes arbitrarily small for $k \rightarrow \infty$. Using a fixed (macroscopic) metric instead, the finite angular resolution amounts to a finite minimal proper distance $L_{\text {min }}^{\text {macro }}$, however.

Acknowledgement: We would like to thank A. Bonanno, O. Lauscher and R. Percacci for helpful discussions. 


\section{References}

[1] For a review see L.J. Garay, Int. J. Mod. Phys. A10 (1995) 145 and references therein.

[2] S. Weinberg in General Relativity, an Einstein Centenary Survey, S.W. Hawking and W. Israel (Eds.), Cambridge University Press (1979);

S. Weinberg, hep-th/9702027.

[3] M. Reuter, Phys. Rev. D 57 (1998) 971 and hep-th/9605030.

[4] D. Dou and R. Percacci, Class. Quant. Grav. 15 (1998) 3449.

[5] O. Lauscher and M. Reuter, Phys. Rev. D 65 (2002) 025013 and hep-th/0108040.

[6] M. Reuter and F. Saueressig, Phys. Rev. D 65 (2002) 065016 and hep-th/0110054

[7] O. Lauscher and M. Reuter, Phys. Rev. D 66 (2002) 025026 and hep-th/0205062.

[8] O. Lauscher and M. Reuter, Class. Quant. Grav. 19 (2002) 483 and hep-th/0110021.

[9] O. Lauscher and M. Reuter, Int. J. Mod. Phys. A 17 (2002) 993 and hep-th/0112089.

[10] W. Souma, Prog. Theor. Phys. 102 (1999) 181.

[11] R. Percacci and D. Perini, Phys. Rev. D 67 (2003) 081503.

[12] R. Percacci and D. Perini, Phys. Rev. D 68 (2003) 044018.

[13] D. Perini, Nucl. Phys. Proc. Suppl. 127 C (2004) 185. 
[14] M. Reuter and F. Saueressig, Phys. Rev. D 66 (2002) 125001 and hep-th/0206145 Fortschr. Phys. 52 (2004) 650 and hep-th/0311056.

[15] D. Litim, Phys. Rev. Lett. 92 (2004) 201301.

[16] A. Bonanno, M. Reuter, JHEP 02 (2005) 035 and hep-th/0410191.

[17] R. Percacci and D. Perini, hep-th/0401071

[18] R. Percacci, hep-th/0409199,

[19] P. Forgács and M. Niedermaier, hep-th/0207028,

M. Niedermaier, JHEP 12 (2002) 066; Nucl. Phys. B 673 (2003) 131.

[20] C. Wetterich, Phys. Lett. B 301 (1993) 90.

[21] M. Reuter and C. Wetterich, Nucl. Phys. B 417 (1994) 181, Nucl. Phys. B 427 (1994) 291, Nucl. Phys. B 391 (1993) 147, Nucl. Phys. B 408 (1993) 91; M. Reuter, Phys. Rev. D 53 (1996) 4430, Mod. Phys. Lett. A 12 (1997) 2777.

[22] For a review see: J. Berges, N. Tetradis and C. Wetterich, Phys. Rep. 363 (2002) 223; C. Wetterich, Int. J. Mod. Phys. A 16 (2001) 1951.

[23] O. Lauscher and M. Reuter, JHEP 10 (2005) 050 and hep-th/0508202.

[24] D. ben-Avraham and S. Havlin, Diffusion and reactions in fractals and disordered systems, Cambridge University Press, Cambridge (2004).

[25] J. Ambjørn, J. Jurkiewicz and R. Loll, Phys. Rev. Lett. 93 (2004) 131301.

[26] J. Ambjørn, J. Jurkiewicz and R. Loll, Phys. Lett. B 607 (2005) 205.

[27] J. Ambjørn, J. Jurkiewicz and R. Loll, preprints hep-th/0505113. hep-th/0505154 hep-th/0509010 
[28] H. Kawai, M. Ninomiya, Nucl. Phys. B 336 (1990) 115;

R. Floreanini and R. Percacci, Nucl. Phys. B 436 (1995) 141;

I. Antoniadis, P.O. Mazur and E. Mottola, Phys. Lett. B 444 (1998) 284.

[29] L.F. Abbott, Nucl. Phys. B 185 (1981) 189; B.S. DeWitt, Phys. Rev. 162 (1967) 1195; M.T. Grisaru, P. van Nieuwenhuizen and C.C. Wu, Phys. Rev. D 12 (1975) 3203; D.M. Capper, J.J. Dulwich and M. Ramon Medrano, Nucl. Phys. B 254 (1985) 737; S.L. Adler, Rev. Mod. Phys. 54 (1982) 729.

[30] For a review see: C. Bagnuls and C. Bervillier, Phys. Rep. 348 (2001) 91; T.R. Morris, Prog. Theor. Phys. Suppl. 131 (1998) 395; J. Polonyi, Central Eur. J. Phys. 1 (2004) 1.

[31] M. Reuter and H. Weyer, JCAP 12 (2004) 001 and hep-th/0410119.

[32] B. Mandelbrot, The Fractal Geometry of Nature, Freeman, New York (1983).

[33] M. Rubin and C. Ordonez, J. Math. Phys. 26 (1985) 65;

A. Higuchi, J. Math. Phys. 28 (1987) 1553.

[34] J. Madore, Class. Quant. Grav. 9 (1992) 69.

[35] A. Bonanno and M. Reuter, Phys. Rev. D 62 (2000) 043008 and hep-th/0002196 Phys. Rev. D 60 (1999) 084011 and gr-qc/9811026.

[36] A. Bonanno and M. Reuter, Phys. Rev. D 65 (2002) 043508 and hep-th/0106133 M. Reuter and F. Saueressig, JCAP 09 (2005) 012 and hep-th/0507167.

[37] A. Bonanno and M. Reuter, Phys. Lett. B 527 (2002) 9 and astro-ph/0106468 Int. J. Mod. Phys. D 13 (2004) 107 and astro-ph/0210472. 
[38] E. Bentivegna, A. Bonanno and M. Reuter, JCAP 01 (2004) 001 and astro-ph/0303150.

[39] A. Bonanno, G. Esposito and C. Rubano, Gen. Rel. Grav. 35 (2003) 1899; Class. Quant. Grav. 21 (2004) 5005; A. Bonanno, G. Esposito, C. Rubano and P. Scudellaro, astro-ph/0507670.

[40] M. Reuter and H. Weyer, Phys. Rev. D 69 (2004) 104022 and hep-th/0311196

[41] M. Reuter and H. Weyer, Phys. Rev. D 70 (2004) 124028 and hep-th/0410117.

[42] J. Moffat, JCAP 05 (2005) 003 and astro-ph/0412195 J.R. Brownstein and J. Moffat, astro-ph/0506370 and astro-ph/0507222. 